\title{
EXISTENCE RESULTS FOR SOME PARTIAL INTEGRODIFFERENTIAL EQUATIONS WITH NONLOCAL CONDITIONS
}

\author{
Khalil Ezzinbi, Saifeddine Ghnimi and Mohamed-Aziz Taoudi \\ Cadi Ayyad University, Morocco and University of Gafsa, Tunisia
}

\begin{abstract}
In this work, we study the existence of mild solutions for a class of partial integrodifferential equations with nonlocal conditions. Our analysis uses the resolvent operator theory and relies on a new fixed point theorem of Sadovskii-Krasnosel'skii type. Our results improve and complement several earlier related works. Some examples are provided to illustrate the theoretical results.
\end{abstract}

\section{INTRODUCTION}

In this work, we study the existence of mild solutions to the following partial integrodifferential equation with nonlocal conditions:

$$
\left\{\begin{array}{l}
u^{\prime}(t)=A u(t)+\int_{0}^{t} B(t-s) u(s) d s+f(t, u(t)) \quad \text { for } t \in[0, a], \\
u(0)=u_{0}+g(u),
\end{array}\right.
$$

where $A: D(A) \subset X \rightarrow X$ is a closed linear operator on a Banach space $(X,||),.(B(t))_{t>0}$ is a family of closed linear operators on $X$ having the same domain $D(B) \supset D(A)$ which is independent of $t, f:[0, a] \times X \rightarrow X$ and $g: C([0, a] ; X) \rightarrow X$ are given functions satisfying conditions to be specified later; here $C([0, a] ; X)$ stands for the space of continuous functions from $[0, a]$ to $X$ endowed with the uniform norm topology.

Equations of the form (1.1) serve as an abstract formulation of many partial integrodifferential equations arising in heat flow in materials with memory, viscoelasticity and many other physical phenomena (see [30,7,28,29] and the

2010 Mathematics Subject Classification. 45K05, 47H10, 47D06.

Key words and phrases. Partial integrodifferential equation, nonlocal conditions, mild solution, fixed point theorem, resolvent operator, semigroup operator. 
references therein). As a concrete special case of (1.1) we can consider the partial neutral differential equation concerning the heat conduction in fading memory material. In the classical theory of heat conduction, it is assumed that the internal energy and the heat flux depend linearly on the temperature $u$ and its gradient $\nabla u$. Under those conditions, the classical heat equation describes sufficiently well the evolution of the temperature in different types of materials. However, this description is not satisfactory in materials with fading memory. In the theory developed in [22,33], the internal energy and the heat flux are described as functionals of $u$ and $u_{x}$. The following system $[7,28,23,12]$ has been frequently used to describe this phenomena,

$\frac{\partial}{\partial t}\left(u(t, x)+\int_{-\infty}^{t} k_{1}(t-s) u(s, x) d s\right)=c \triangle u(t, x)+\int_{-\infty}^{t} k_{2}(t-s) \triangle u(s, x) d s$.

Here, $\Omega \subset \mathbb{R}^{n}$ is open, bounded and has smooth boundary, $(t ; x) \in[0 ; \infty) \times \Omega$, $u(t ; x)$ represents the temperature in $x$ at the time $t, c$ is a physical constant and $k_{i}: \mathbb{R} \rightarrow \mathbb{R}, i=1,2$, are the internal energy and the heat flux relaxation respectively. By assuming that the solution $u($.$) is known on (-\infty ; 0]$ and $k_{1} \equiv 0$ we can transform this system into the abstract form (1.1), with $A=\triangle$ and $B(t)=k_{2}(t) \triangle$.

Another special case of (1.1) is the following model

$\frac{d}{d t}\left(u(t)-\lambda \int_{-\infty}^{t} C(t-s) u(s) d s\right)=A u(t)+\lambda \int_{-\infty}^{t} B(t-s) u(s) d s-p(t)+q(t)$,

which arises in the dynamics of income, employment, value of capital stock, and cumulative balance of payment, see [9] for more details. Here $\lambda$ is a real number, the state $u(t) \in \mathbb{R}^{n}, C($.$) and B($.$) are n \times n$ continuous function matrices, $A$ is a constant $n \times n$ matrix, $p(t)$ represents the government intervention, and $q(t)$ the private initiative. If the solution $u$ is known on $(-\infty, 0]$ and $C \equiv 0$, then this system can be transformed into an abstract system described as (1.1).

The problem of existence and uniqueness of solutions of equations of the special form as in (1.1) have been studied by many authors using different approaches. Specifically, there exists an extensive literature related to the existence and qualitative properties of integrodifferential equations using resolvent operator technique. We refer the reader to the book by Gripenberg et al. ([21]) which contains an overview of the theory in finite dimensional case. For abstract integrodifferential equations described on infinite dimensional spaces, we refer the reader to the monograph by Pruss ([34]).

In some phenomena, nonlocal initial conditions are more realistic and practical than the classical ones in handling physical problems. As stressed in $[3,11]$ and the references therein, the nonlocal condition $u(0)=u_{0}+g(u)$ has better effect in some physical problems than the classical condition $u(0)=u_{0}$. 
For example, in [11], the author introduced the following nonlocal condition:

$$
g(u)=\sum_{i=1}^{n} c_{i} u\left(t_{i}\right),
$$

where $c_{i}, i=1,2, \cdots, n$ and $0<t_{1}<t_{2}<\cdots<a$, to describe the diffusion phenomenon of a small amount of gas in a transparent tube. In this case, the above explanation allows the additional measurements at $t_{i}, i=1,2, \cdots, n$.

The study of the existence of solutions for some evolution equations with nonlocal conditions was initiated by Byszewski in [3]. The authors proved the existence and uniqueness of mild, strong and classical solutions of (1.1), when $B=0$. Since then, the problem was addressed by many investigators. We quote the contributions by Byszewski et al. ([4-6]), Ezzinbi and Fu ([16]), Ezzinbi et al. ([14,17,19,15]), Liang, Liu and Xia ([24]), Ntouyas and Tsamatos ([32]), Zhu et al. ([36]) and many others.

Recently, Lizama et al. in [27] proved the existence of mild solutions to the nonlocal problem (1.1) when $g$ is compact and the resolvent operator $R(t)$ is norm continuous. The analysis uses the resolvent operator theory and Sadovskii's fixed point theorem.

In this work, we propose a new approach, to avoid the compactness of $g$, which combines iterative techniques with a new fixed point theorem of Sadovskii-Krasnosel'skii type recently proved in [18]. We also prove that the immediate norm continuity of the resolvent operator $R(t)$ associated to (1.1) is equivalent to the immediate norm continuity of the semigroup $T(t)$ generated by $A$. This result is interesting in itself and is of fundamental importance from practical viewpoint. In fact, in many applications it is always not possible to show that the resolvent family associated to (1.1) is immediately norm continuous, while the immediate norm continuity of the semigroup generated by $A$ offers no problem.

The outline of this work is as follows. In Section 2, we recall some basic definitions and preliminary facts which will be used throughout this work. In Section 3, we first give necessary and sufficient conditions for the norm continuity of the resolvent operator associated to (1.1). Next we prove the existence of mild solutions of (1.1) under rather general and nonrestrictive assumptions. In Section 4, we provide two examples to illustrate the theoretical results.

\section{Preliminaries}

In this section, we introduce some definitions and preliminary facts which are used in this work. Let $Z$ and $W$ be Banach spaces. We denote by $\mathcal{L}(Z, W)$ the Banach space of bounded linear operators from $Z$ into $W$ endowed with the operator norm and we abbreviate this notation to $\mathcal{L}(Z)$ when $Z=W$. We now recall an important fact on the resolvent operator theory, see $[8,10,20]$. 
Let us consider the following integrodifferential equation:

$$
\left\{\begin{array}{l}
y^{\prime}(t)=A y(t)+\int_{0}^{t} B(t-s) y(s) d s \text { for } t \geq 0 \\
y(0)=y_{0} \in X
\end{array}\right.
$$

In the sequel, we assume that:

(H1) $A$ is a closed densely defined linear operator on a Banach space $(X,||$.$) .$ Since $A$ is closed, then $D(A)$ equipped with the graph norm $\|x\|:=$ $|A x|+|x|$ is a Banach space which is denoted by $(Y,\|\cdot\|)$.

(H2) $(B(t))_{t>0}$ is a family of linear operators on $X$ such that $B(t)$ is continuous from $Y$ to $X$ for almost all $t \geq 0$. Moreover, there is a locally integrable function $b: \mathbb{R}^{+} \rightarrow \mathbb{R}^{+}$such that $B(t) y$ is measurable and $|B(t) y| \leq b(t)\|y\|$ for all $y \in Y$ and $t \geq 0$.

(H3) For any $y \in Y$, the map $t \rightarrow B(t) y$ belongs to $W_{l o c}^{1,1}\left(\mathbb{R}^{+}, X\right)$ and

$$
\left|\frac{d}{d t} B(t) y\right| \leq b(t)\|y\| \text { for } y \in Y \text { and a.e. } t \in \mathbb{R}^{+} \text {. }
$$

Definition 2.1 ([20]). A resolvent operator for (2.1) is a bounded linear operator valued function $R(t) \in \mathcal{L}(X)$ for $t \geq 0$ having the following properties:

(a) $R(0)=I$, the identity map on $X$ and $|R(t)| \leq M e^{\beta t}$ for some constants $M$ and $\beta$.

(b) For each $x \in X, R(t) x$ is strongly continuous for $t \geq 0$.

(c) $R(t) \in \mathcal{L}(Y)$ for $t \geq 0$. For $x \in Y, R(). x \in C^{1}\left(\mathbb{R}^{+} ; X\right) \cap C\left(\mathbb{R}^{+} ; Y\right)$ and

$$
\begin{aligned}
R^{\prime}(t) x & =A R(t) x+\int_{0}^{t} B(t-s) R(s) x d s \\
& =R(t) A x+\int_{0}^{t} R(t-s) B(s) x d s \text { for } t \geq 0 .
\end{aligned}
$$

The following result provides sufficient conditions for the existence of the resolvent operator for $(2.1)$.

Theorem 2.2 ([10]). Assume that (H1)-(H3) hold. Then (2.1) admits a resolvent operator if and only if $A$ generates a $C_{0}$-semigroup.

From now on, we will assume that:

(H4) $A$ generates a $C_{0}$-semigroup $(T(t))_{t \geq 0}$.

Before to resume our analysis, let us recall some relevant definitions and results. The Kuratowski measure of noncompactness is defined by:

$$
\begin{gathered}
\alpha(B)=\inf \{d>0 ; B \text { can be covered by a finite number } \\
\text { of sets of diameter }<d\},
\end{gathered}
$$

for each bounded subset $B$ of $X$. The following lemmas summarize several properties of $\alpha($.$) that are useful for our purposes.$ 
Lemma 2.3 ([1]). Let $B, C \subseteq X$ be bounded, Then

(1) $\alpha(B)=0$ if and only if $B$ is relatively compact;

(2) $\alpha(B)=\alpha(\bar{B})=\alpha(\overline{c o} B)$, where $\overline{c o} B$ is the closed convex hull of $B$;

(3) $\alpha(B) \leq \alpha(C)$ when $B \subseteq C$;

(4) $\alpha(B+C) \leq \alpha(B)+\alpha(C)$;

(5) $\alpha(B \cup C) \leq \max \{\alpha(B), \alpha(C)\}$;

(6) $\alpha(B(0, r)) \leq 2 r$, where $B(0, r)=\{x \in X:|x| \leq r\}$.

By a measure of noncompactness on a Banach space $X$ we mean a map $\psi: \mathcal{B}(X) \rightarrow \mathbb{R}_{+}$which satisfies conditions (1)-(5) in Lemma 2.3 ; where $\mathcal{B}(X)$ stands for the collection of bounded subsets of $X$.

For later use, we recall the following lemmas.

Lemma 2.4 ([1]). Let $G: X \longrightarrow X$ be a Lipschitz continuous map with constant $k$. Then $\alpha(G(B)) \leq k \alpha(B)$ for any bounded subset $B$ of $X$.

Lemma 2.5 ([35]). Let $\mathcal{H} \subseteq C([0, a], X)$ be equicontinuous and $x_{0} \in$ $C([0, a], X)$. Then $\overline{c o}\left(\mathcal{H} \cup\left\{x_{0}\right\}\right)$ is also equicontinuous in $C([0, a], X)$.

We shall make extensive use of the following lemma.

Lemma $2.6([1])$. Let $\mathcal{H} \subset C([0, a] ; X)$ be a bounded set. Then $\alpha(\mathcal{H}(t)) \leq$ $\alpha(\mathcal{H})$ for any $t \in[0, a]$, where $\mathcal{H}(t)=\{u(t): u \in \mathcal{H}\}$. Furthermore if $\mathcal{H}$ is equicontinuous on $[0, a]$, then $t \longmapsto \alpha(\mathcal{H}(t))$ is continuous on $[0, a]$ and

$$
\alpha(\mathcal{H})=\sup \{\alpha(\mathcal{H}(t)): t \in[0, a]\} .
$$

Definition 2.7. A set of functions $\mathcal{H} \subseteq L^{1}([0, a] ; X)$ is said to be uniformly integrable if there exists a positive function $\kappa \in L^{1}\left([0, a] ; \mathbb{R}^{+}\right)$such that $|h(t)| \leq \kappa(t)$ a.e. for all $h \in \mathcal{H}$.

Lemma 2.8 ([31]). If $\left\{u_{n}\right\}_{n \in \mathbb{N}} \subseteq L^{1}([0, a] ; X)$ is uniformly integrable, then for each $n \in \mathbb{N}$ the function $t \mapsto \alpha\left(\left\{u_{n}\right\}_{n \in \mathbb{N}}\right)$ is measurable and

$$
\alpha\left(\left\{\int_{0}^{t} u_{n}(s) d s\right\}_{n=1}^{\infty}\right) \leq 2 \int_{0}^{t} \alpha\left(\left\{u_{n}(s)\right\}_{n=1}^{\infty}\right) d s .
$$

Lemma 2.9 ([2]). Let $\mathcal{H}$ be a bounded subset of $X$. Then for each $\varepsilon>0$, there exists a sequence $\left\{u_{n}\right\}_{n \in \mathbb{N}} \subseteq \mathcal{H}$ such that

$$
\alpha(\mathcal{H}) \leq 2 \alpha\left(\left\{u_{n}\right\}_{n=1}^{\infty}\right)+\varepsilon .
$$

We also need the following elementary result.

Lemma 2.10 ([26]). For all $0 \leq m \leq n$, we denote $C_{n}^{m}=\left(\begin{array}{c}m \\ n\end{array}\right)$. Let $0<\epsilon<1, h>0$ and

$$
S_{n}=\epsilon^{n}+C_{n}^{1} \epsilon^{n-1} h+C_{n}^{2} \epsilon^{n-2} \frac{h^{2}}{2 !}+\ldots+\frac{h^{n}}{n !}, n \in \mathbb{N} .
$$

Then, $\lim _{n \rightarrow \infty} S_{n}=0$. 
Now, we make a short note on convex-power condensing operators. Let $M$ be a nonempty closed convex subset of $X, K, S: M \rightarrow X$ two nonlinear mappings and $x_{0} \in X$. For any $D \subseteq M$, we define

$$
\mathcal{F}^{\left(1, x_{0}\right)}(K, S, D)=\{x \in M: x=S x+K y \text {, for some } y \in D\},
$$

and

$$
\mathcal{F}^{\left(n, x_{0}\right)}(K, S, D)=\mathcal{F}^{\left(1, x_{0}\right)}\left(K, S, \overline{c o}\left(\mathcal{F}^{\left(n-1, x_{0}\right)}(K, S, D) \cup\left\{x_{0}\right\}\right)\right),
$$

for $n=2,3, \ldots$.

Definition 2.11 ([35]). Let $X$ be a Banach space, $M$ be a nonempty closed convex subset of $X$ and $\psi$ a measure of noncompactness on $X$. Let $K, S: M \rightarrow X$ be two bounded mappings (i.e. they take bounded sets into bounded ones) and $x_{0} \in M$. We say that $K$ is a $S$-convex-power condensing operator about $x_{0}$ and $n_{0}$ w.r.t. $\psi$ if for any bounded set $D \subseteq M$ with $\psi(D)>$ 0 we have

$$
\psi\left(\mathcal{F}^{\left(n_{0}, x_{0}\right)}(K, S, D)\right)<\psi(D) .
$$

The considerations of this paper are based on the following fixed point result.

TheOrem 2.12 ([18]). Let $X$ be a Banach space and $\psi$ be a measure of noncompactness on $X$. Let $M$ be a nonempty bounded closed convex subset of $X$. Suppose that $K, S: M \rightarrow X$ are two continuous mappings satisfying:

(i) $S$ is a strict contraction,

(ii) $S x+K y \in M$ for all $x, y \in M$,

(iii) there are an integer $n$ and a vector $x_{0} \in X$ such that $K$ is $S$-powerconvex condensing w.r.t. $\psi$.

Then $S+K$ has at least one fixed point in $M$.

\section{MAin RESUlts}

3.1. Norm continuity of $R($.$) . In this subsection, we prove that the im-$ mediate norm continuity of the resolvent operator $R(t)$ associated to $(2.1)$ is equivalent to the immediate norm continuity of the semigroup $T(t)$ generated by $A$. Before we prove this result, we state the following lemma.

Lemma 3.1 ([10, Lemma 1]). Assume that $(\mathbf{H 1})-(\mathbf{H 4})$ are satisfied. Let $(T(t))_{t \geq 0}$ be the $C_{0}$-semigroup generated by $A$ and $(R(t))_{t \geq 0}$ be the resolvent operator of (2.1). Then

$$
R(t) x=T(t) x+\int_{0}^{t} T(t-s) Q(s) x d s
$$

and

$$
T(t) x=R(t) x+\int_{0}^{t} R(t-s) W(s) x d s
$$


with

$$
Q(s) x=B(0) \int_{0}^{s} R(u) x d u+\int_{0}^{s} B^{\prime}(s-u) \int_{0}^{u} R(v) x d v d u
$$

and

$$
W(s) x=-B(0) \int_{0}^{s} T(u) x d u-\int_{0}^{s} B^{\prime}(s-u) \int_{0}^{u} T(v) x d v d u .
$$

The operators $Q($.$) and W($.$) are uniformly bounded on bounded intervals,$ that is, for each $a>0$ there exists $k(a)$ and $k^{\prime}(a)$ such that $|Q(s)| \leq k(a)$ and $|W(s)| \leq k^{\prime}(a)$ for $0 \leq s \leq a$. Moreover, for each $x \in X, Q()$.$x and W()$. belong to $C([0, \infty), X)$.

Now, we are ready to state the following result.

Theorem 3.2. Assume that $(\mathbf{H 1})-(\mathbf{H 4})$ are satisfied. Then $(T(t))_{t \geq 0}$ is norm continuous (or continuous in the uniform operator topology) for $t>0$ if and only if the corresponding resolvent operator $(R(t))_{t \geq 0}$ of (2.1) is norm continuous for $t>0$.

Proof. The proof is reasonably straightforward using Lemma 3.1.

3.2. Existence result. In this subsection, we prove the existence of a mild solution for the nonlocal problem (1.1). By a mild solution of (1.1) we mean a continuous function $u:[0, a] \rightarrow X$ satisfying:

$$
u(t)=R(t)\left(u_{0}+g(u)\right)+\int_{0}^{t} R(t-s) f(s, u(s)) d s \text { for } t \in[0, a] .
$$

To ensure that a mild solution of (1.1) exists, we make the following assumptions.

(H5) The semigroup $(T(t))_{t \geq 0}$ is norm continuous for $t>0$.

(H6) The function $f:[0, a] \times X \rightarrow X$ satisfies the Carathéodory conditions; that is, $f(., u)$ is measurable for all $u \in X$ and $f(t,$.$) is continuous for$ almost all $t \in[0, a]$.

(H7) There exist a function $\rho \in L^{1}\left([0, a] ; \mathbb{R}^{+}\right)$and a nondecreasing continuous function $\Omega: \mathbb{R}^{+} \rightarrow \mathbb{R}^{+}$such that

$$
|f(t, u)| \leq \rho(t) \Omega(|u|) \text { for all } t \in[0, a] \text { and } u \in X .
$$

(H8) There exists a function $C \in L^{1}\left([0, a] ; \mathbb{R}^{+}\right)$such that for any bounded set $D \subseteq X$

$$
\alpha(f(t, D)) \leq C(t) \alpha(D) .
$$

(H9) There exists a nonnegative constant $L_{g}$, such that

$$
|g(u)-g(v)| \leq L_{g}|u-v| \text { for } u, v \in C([0, a] ; X) .
$$

Now we are in a position to state our existence result. 
Theorem 3.3. Assume that $(\mathbf{H 1})-(\mathbf{H 9})$ hold. Then the nonlocal problem (1.1) has at least one mild solution on $[0, a]$ provided that

$$
M_{a}\left(L_{g}+\int_{0}^{a} \rho(s) d s \liminf _{r \rightarrow \infty} \frac{\Omega(r)}{r}\right)<1
$$

where, $M_{a}=\sup _{0 \leq t \leq a}|R(t)|$.

Proof. To allow the abstract formulation of our problem, we define the operators $S, K: C([0, a] ; X) \rightarrow C([0, a] ; X)$ as follows

$$
\begin{aligned}
& (S u)(t)=R(t)\left(u_{0}+g(u)\right) \text { for } \quad t \in[0, a] \\
& (K v)(t)=\int_{0}^{t} R(t-s) f(s, v(s)) d s \quad \text { for } \quad t \in[0, a] .
\end{aligned}
$$

Then $u$ is a mild solution for (1.1) if and only if $u$ is a fixed point for the sum $S+K$. We shall point out that assumptions of Theorem 2.12 are fulfilled.

First, we show that $K, S$ are continuous on $C([0, a] ; X)$. To see this, let $\left(u_{n}\right)_{n}$ be a sequence in $C([0, a] ; X)$ such that $\lim _{n \rightarrow \infty} u_{n}=u$ in $C([0, a] ; X)$. By assumption (H6) we know that for a.e. $s \in[0, a]$, we have

$$
\lim _{n \rightarrow \infty} f\left(s, u_{n}(s)\right)=f(s, u(s)) \text {. }
$$

Thus

$$
\left|K u_{n}-K u\right| \leq M_{a} \int_{0}^{a}\left|f\left(s, u_{n}(s)\right)-f(s, u(s))\right| d s .
$$

Using the dominated convergence theorem, we deduce that $\lim _{n \rightarrow \infty}\left|K u_{n}-K u\right|=$ 0 . The continuity of $S$ follows from (H9). Now, let $r>0$ and $B_{r}=\{u \in$ $C([0, a] ; X):|u| \leq r\}$. For $u, v \in B_{r}$ and $t \in[0, a]$, we have

$$
\begin{aligned}
|(S u)(t)+(K v)(t)| & \leq\left|R(t)\left(u_{0}+g(u)\right)\right|+\left|\int_{0}^{t} R(t-s) f(s, v(s)) d s\right| \\
& \leq M_{a}\left(\left|u_{0}\right|+|g(0)|+r L_{g}\right)+M_{a} \Omega(r) \int_{0}^{a} \rho(s) d s .
\end{aligned}
$$

We show that there exists $r>0$ such that $S u+K v \in B_{r}$. If it is not the case, then for each $r>0$ there exists $u, v \in B_{r}$ such that $S u+K v \notin B_{r}$, that is,

$$
r<|(S u)+(K v)| \leq M_{a}\left(\left|u_{0}\right|+|g(0)|+r L_{g}\right)+M_{a} \Omega(r) \int_{0}^{a} \rho(s) d s,
$$

which implies when dividing by $r$ that

$$
1<\frac{M_{a}}{r}\left(\left|u_{0}\right|+|g(0)|\right)+M_{a} L_{g}+\frac{M_{a}}{r} \Omega(r) \int_{0}^{a} \rho(s) d s .
$$

Taking liminf as $r \rightarrow \infty$, we obtain

$$
1 \leq M_{a}\left(L_{g}+\int_{0}^{a} \rho(s) d s \liminf _{r \rightarrow \infty} \frac{\Omega(r)}{r}\right)
$$


which contradicts the assumption (3.4). Therefore, there exists $r_{0}>0$ such that

$$
|(S u)+(K v)| \leq r_{0} .
$$

Thus $S u+K v \in B_{r_{0}}$ for all $u, v \in B_{r_{0}}$. Next, we claim that the operator $S$ is a strict contraction. Indeed, by $(\mathbf{H 9})$ we can see that

$$
|(S u)(t)-(S v)(t)| \leq|R(t)||(g(u)-g(v))| \leq M_{a} L_{g}|u-v| .
$$

for any $u, v \in B_{r_{0}}$ and any $t \in[0, a]$. Consequently,

$$
|S u-S v| \leq k|u-v|,
$$

where $k=M_{a} L_{g}<1$. This proves our claim.

Finally, we prove that the operator $K$ is $S$-power-convex condensing. This will be achieved in three steps. Let $D$ be a subset of $B_{r_{0}}$.

SteP 1. We show that $K(D)=\left\{K u=\int_{0}^{t} R(t-s) f(s, u(s)) d s: u \in D\right\}$ is equicontinuous on $[0, a]$. To see this, let $t=0$ and $t^{\prime}>0$. Then from $(\mathbf{H 7})$ we have

$$
\left|(K u)\left(t^{\prime}\right)-(K u)(0)\right| \leq \int_{0}^{t^{\prime}}\left|R\left(t^{\prime}-s\right)\right||f(s, u(s))| d s \leq M_{a} \Omega(r) \int_{0}^{t^{\prime}} \rho(s) d s .
$$

Since $\rho \in L^{1}\left([0, a], \mathbb{R}^{+}\right)$then $\left|(K u)\left(t^{\prime}\right)-(K u)(0)\right|$ tends to zero independently of $u \in D$ as $t^{\prime}$ tends to zero. Hence $K(D)$ is equicontinuous at $t=0$.

Let $0<t<t^{\prime} \leq a$. Then

$$
\begin{aligned}
\left|(T u)\left(t^{\prime}\right)-(T u)(t)\right| \leq & \int_{t}^{t^{\prime}}\left|R\left(t^{\prime}-s\right)\right||f(s, u(s))| d s \\
& +\int_{0}^{t}\left|\left(R\left(t^{\prime}-s\right)-R(t-s)\right)\right||f(s, u(s))| d s .
\end{aligned}
$$

Using Theorem 3.2 and (H5) we deduce that

$$
\left|R\left(t^{\prime}-s\right)-R(t-s)\right| \rightarrow 0 \text { as } t^{\prime} \rightarrow t \text { almost all } s \neq t .
$$

Since the function $s \mapsto R\left(t^{\prime}-s\right) f(s, u(s))$ is $L^{1}\left([0, a] ; \mathbb{R}^{+}\right)$, then the Lebesgue dominated convergence theorem implies that

$$
\int_{0}^{t}\left|R\left(t^{\prime}-s\right)-R(t-s)\right| \mid f\left(s, u(s) \mid d s \rightarrow 0 \text { as } t^{\prime} \rightarrow t .\right.
$$

Moreover,

$\int_{0}^{t}\left|R\left(t^{\prime}-s\right)-R(t-s)\right| \mid f\left(s, u(s)\left|d s \leq \Omega(r) \int_{0}^{t}\right| R\left(t^{\prime}-s\right)-R(t-s) \mid \rho(s) d s\right.$.

Therefore,

$$
\sup _{u \in D} \int_{0}^{t}\left|R\left(t^{\prime}-s\right)-R(t-s)\right| \mid f\left(s, u(s) \mid d s \rightarrow 0 \text { as } t^{\prime} \rightarrow t .\right.
$$

Consequently, $K(D)$ is equicontinuous on $[0, a]$. 
SteP 2. We then illustrate that $\mathcal{F}^{(n, 0)}(K, S, D)$ is equicontinuous on $[0, a]$ for any integer $n \geq 1$. To perform this, notice first that for $u \in \mathcal{F}^{(1,0)}(K, S, D)$ there exists $v \in D$ such that $u=S u+K v$. Then for $t, t^{\prime} \in[0, a]$ we have

$$
\begin{aligned}
\left|u(t)-u\left(t^{\prime}\right)\right| & =\left|S u(t)+K v(t)-S u\left(t^{\prime}\right)-K v\left(t^{\prime}\right)\right| \\
& \leq\left|S u(t)-S u\left(t^{\prime}\right)\right|+\left|K v(t)-K v\left(t^{\prime}\right)\right| \\
& \leq k\left|u(t)-u\left(t^{\prime}\right)\right|+\left|K v(t)-K v\left(t^{\prime}\right)\right| .
\end{aligned}
$$

Hence,

$$
\left|u(t)-u\left(t^{\prime}\right)\right| \leq \frac{1}{1-k}\left|K v(t)-K v\left(t^{\prime}\right)\right| .
$$

Now the equicontinuity of $\mathcal{F}^{(1,0)}(K, S, D)$ follows from Step 1 . The same reasoning as above implies that $\mathcal{F}^{(2,0)}(K, S, D):=\mathcal{F}^{(1,0)}\left(K, S, \overline{c o}\left(\mathcal{F}^{(1,0)}(K, S, D)\right.\right.$ $\cup\{0\}$ ), is equicontinuous. By mathematical induction we can prove that $\mathcal{F}^{(n, 0)}(K, S, D)$ is equicontinuous for all $n \geq 1$.

STEP 3. We show that there is an integer $n_{0}$ such that $\alpha\left(\mathcal{F}^{\left(n_{0}, 0\right)}(K, S, D)\right)$ $<\alpha(D)$. Notice that for $t \in[0, a]$, we have

$$
\begin{aligned}
\mathcal{F}^{(1,0)}(K, S, D)(t)= & \left\{u(t), u \in \mathcal{F}^{(1,0)}(K, S, D)\right\} \\
\subseteq & \left\{u(t)-S u(t), u \in \mathcal{F}^{(1,0)}(K, S, D)\right\} \\
& +\left\{S u(t), u \in \mathcal{F}^{(1,0)}(K, S, D)\right\} .
\end{aligned}
$$

By using the properties of the measure of noncompactness and properties of $S$, we get

$$
\alpha\left(\mathcal{F}^{(1,0)}(K, S, D)(t)\right) \leq \alpha(K(D)(t))+k \alpha\left(\mathcal{F}^{(1,0)}(K, S, D)(t)\right) .
$$

This implies that

$$
\alpha\left(\mathcal{F}^{(1,0)}(K, S, D)(t)\right) \leq \frac{1}{1-k} \alpha(K(D)(t))
$$

On the other hand since $K(D)$ is bounded, then from Lemma 2.9 for each $\varepsilon>0$, there exists a sequence $\left\{v_{n}\right\}_{n \in \mathbb{N}} \subseteq K(D)$ such that

$$
\alpha(K(D)(t)) \leq 2 \alpha\left(\left\{v_{n}(t)\right\}_{n=1}^{\infty}\right)+\varepsilon \leq 2 \alpha\left(\left\{\int_{0}^{t} R(t-s) f\left(s, u_{n}(s)\right) d s\right\}_{n=1}^{\infty}\right)+\varepsilon .
$$

Since $\left|R(t-s) f\left(s, u_{n}(s)\right)\right| \leq M_{a} \Omega(r) \rho(s)$ and $\rho \in L^{1}\left([0, a] ; \mathbb{R}^{+}\right)$, then Lemma 2.8 implies that

$$
\alpha(K(D)(t)) \leq 4 M_{a} \int_{0}^{t} \alpha\left(\left\{f\left(s, u_{n}(s)\right)\right\}_{n=1}^{\infty}\right) d s+\varepsilon .
$$

Thus in virtue of (H8) we have

$$
\alpha(K(D)(t)) \leq 4 M_{a} \int_{0}^{t} C(s) \alpha\left(\left\{u_{n}(s)\right\}_{n \in \mathbb{N}}\right) d s+\varepsilon \leq 4 M_{a} \alpha(D) \int_{0}^{t} C(s) d s+\varepsilon .
$$


From the density of $C\left([0, a] ; \mathbb{R}^{+}\right)$in $L^{1}\left([0, a] ; \mathbb{R}^{+}\right)$and since $C \in L^{1}\left([0, a] ; \mathbb{R}^{+}\right)$, then for $\delta<\frac{1-k}{4 M_{a}}$ there exists $\varphi \in C\left([0, a] ; \mathbb{R}^{+}\right)$satisfying $\int_{0}^{a}|C(s)-\varphi(s)| d s<$ $\delta$. Consequently

$$
\begin{aligned}
\alpha(K(D)(t)) & \leq 4 M_{a} \alpha(D)\left[\int_{0}^{t}|C(s)-\varphi(s)| d s+\int_{0}^{t}|\varphi(s)| d s\right]+\varepsilon \\
& \leq 4 M_{a} \alpha(D)[\delta+\tau t]+\varepsilon,
\end{aligned}
$$

where $\tau=\sup _{0 \leq s \leq a}|\varphi(s)|$. Letting $\varepsilon \rightarrow 0$, we get

$$
\alpha(K(D)(t)) \leq\left(4 M_{a} \delta+4 M_{a} \tau t\right) \alpha(D) .
$$

This means by (3.5) that

$$
\alpha\left(\mathcal{F}^{(1,0)}(K, S, D)(t)\right) \leq(\lambda+\mu t) \alpha(D),
$$

where $\lambda=\frac{4 M_{a} \delta}{(1-k)}$ and $\mu=\frac{4 M_{a} \tau}{(1-k)}$. On the other hand,

$$
\begin{aligned}
\mathcal{F}^{(2,0)}(K, S, D)(t) \subseteq & \left\{u(t)-S u(t), u \in \mathcal{F}^{(2,0)}(K, S, D)\right\} \\
& +\left\{S u(t), u \in \mathcal{F}^{(2,0)}(K, S, D)\right\} \\
\subseteq & \left\{K v(t), v \in \overline{c o}\left(\mathcal{F}^{(1,0)}(K, S, D) \cup\{0\}\right)\right\} \\
& +\left\{S u(t), u \in \mathcal{F}^{(2,0)}(K, S, D)\right\} .
\end{aligned}
$$

Referring to Lemmas 2.3 and 2.4, we see that

$$
\begin{aligned}
\alpha\left(\mathcal{F}^{(2,0)}(K, S, D)(t)\right) \leq & \alpha\left(K\left(\overline{c o}\left(\mathcal{F}^{(1,0)}(K, S, D) \cup\{0\}\right)\right)(t)\right) \\
& +k \alpha\left(\mathcal{F}^{(2,0)}(K, S, D)(t)\right) .
\end{aligned}
$$

Thus

$$
\alpha\left(\mathcal{F}^{(2,0)}(K, S, D)(t)\right) \leq \frac{1}{1-k} \alpha\left(K\left(\overline{c o}\left(\mathcal{F}^{(1,0)}(K, S, D) \cup\{0\}\right)\right)(t)\right) .
$$

Moreover from Lemma 2.9 there exists a sequence $\left\{w_{n}\right\}_{n \in \mathbb{N}} \subseteq \overline{c o}\left(\mathcal{F}^{(1,0)}(K, S, D)\right.$ $\cup\{0\})$ such that

$$
\begin{aligned}
\alpha\left(K\left(\overline{c o}\left(\mathcal{F}^{(1,0)}(K, S, D) \cup\{0\}\right)\right)(t)\right) \\
\quad \leq 2 \alpha\left(\left\{\int_{0}^{t} R(t-s) f\left(s, w_{n}(s)\right) d s\right\}_{n=1}^{\infty}\right)+\varepsilon \\
\quad \leq 4 M_{a} \int_{0}^{t} \alpha\left(\left\{f\left(s, w_{n}(s)\right)\right\}_{n=1}^{\infty}\right) d s+\varepsilon \\
\quad \leq 4 M_{a} \int_{0}^{t} C(s) \alpha\left(\overline{c o}\left(\mathcal{F}^{(1,0)}(K, S, D) \cup\{0\}\right)(s)\right) d s+\varepsilon \\
\quad \leq 4 M_{a} \int_{0}^{t} C(s) \alpha\left(\mathcal{F}^{(1,0)}(K, S, D)(s)\right) d s+\varepsilon .
\end{aligned}
$$


Linking (3.6) and (3.7) we get

$$
\begin{aligned}
\alpha\left(\mathcal{F}^{(2,0)}(K, S, D)(t)\right) \\
\quad \leq \frac{4 M_{a}}{(1-k)} \int_{0}^{t}[|C(s)-\varphi(s)|+|\varphi(s)|](\lambda+\mu s) \alpha(D) d s+\frac{\varepsilon}{(1-k)} \\
\quad \leq \frac{4 M_{a}}{(1-k)}\left[(\lambda+\mu t) \int_{0}^{t}|C(s)-\varphi(s)| d s+\tau\left(\lambda t+\mu \frac{t^{2}}{2}\right)\right] \alpha(D)+\frac{\varepsilon}{(1-k)} \\
\quad \leq\left[\lambda(\lambda+\mu t)+\mu\left(\lambda t+\mu \frac{t^{2}}{2}\right)\right] \alpha(D)+\frac{\varepsilon}{(1-k)} \\
\quad \leq\left[\lambda^{2}+2 \lambda \mu t+\frac{(\mu t)^{2}}{2}\right] \alpha(D)+\frac{\varepsilon}{(1-k)} .
\end{aligned}
$$

Letting $\varepsilon \rightarrow 0$, we obtain

$$
\alpha\left(\mathcal{F}^{(2,0)}(K, S, D)(t)\right) \leq\left[\lambda^{2}+2 \lambda \mu t+\frac{(\mu t)^{2}}{2}\right] \alpha(D) .
$$

By mathematical induction we obtain that for all integer $n \geq 1$

$\left.\alpha\left(\mathcal{F}^{(n, 0)}(K, S, D)(t)\right) \leq\left[\lambda^{n}+C_{n}^{1} \lambda^{n-1} \mu t+C_{n}^{2} \lambda^{n-2} \frac{(\mu t)^{2}}{2 !}\right)+\ldots+\frac{(\mu t)^{n}}{n !}\right] \alpha(D)$.

Using the equicontinuity of $\mathcal{F}^{(n, 0)}(K, S, D)$ together with Lemma 2.6, we infer that

$\left.\alpha\left(\mathcal{F}^{(n, 0)}(K, S, D)\right) \leq\left[\lambda^{n}+C_{n}^{1} \lambda^{n-1} \mu a+C_{n}^{2} \lambda^{n-2} \frac{(\mu a)^{2}}{2 !}\right)+\ldots+\frac{(\mu a)^{n}}{n !}\right] \alpha(D)$.

As $0<\lambda<1$ and $\mu a>0$, it follows from Lemma 2.10 that there exists $n_{0} \in \mathbb{N}$ such that

$$
\left.S_{n_{0}}=\left[\lambda^{n_{0}}+C_{n_{0}}^{1} \lambda^{n_{0}-1} \mu a+C_{n_{0}}^{2} \lambda^{n_{0}-2} \frac{(\mu a)^{2}}{2 !}\right)+\ldots+\frac{(\mu a)^{n_{0}}}{n_{0} !}\right]<1 .
$$

This implies

$$
\alpha\left(\mathcal{F}^{\left(n_{0}, 0\right)}(K, S, D)\right)<\alpha(D) .
$$

Consequently, $K$ is $S$-power-convex condensing. Applying Theorem 2.12, we deduce that $S+K$ has at least one fixed point in $B_{r_{0}}$ which is, in turn, a mild solution of (1.1).

Remark 3.4. Theorem 3.3 extends [27, Theorem 3.2] and [36, Theorem 3.1]. In [27] $g$ is assumed to be compact and the resolvent operator is immediately norm continuous. In [36], the compactness of $g$ and $B=0$ are required. 


\section{Applications}

4.1. Application 1. To apply our results, we consider the following nonlocal partial integrodifferential equation

(4.1)

$$
\left\{\begin{array}{l}
\frac{\partial}{\partial t} w(t, x)=q(x) w(t, x)+\int_{0}^{t} b(t-s) q(x) w(s, x) d s+f_{1}(t) f_{2}(w(t, x)) \\
w(t, 0)=w(t, 1)=0 \text { for } t \in[0, a], \\
w(0, x)=w_{0}(x)+G(w(t, x)) \text { for } t \in[0, a] \text { and } x \in[0, a] \text { and } x \in[0,1]
\end{array}\right.
$$

Let $X=C_{0}([0,1] ; \mathbb{C})$ be the space of continuous functions from $[0,1]$ to $\mathbb{C}$ vanishing at 0 and 1 endowed with the uniform norm topology. We assume that:

(i) $w_{0} \in C_{0}([0,1] ; \mathbb{C})$.

(ii) $b: \mathbb{R}_{+} \rightarrow \mathbb{R}_{+}$is a $\mathcal{C}^{1}$ function with a derivative $\left|b^{\prime}(t)\right| \leq b(t)$ for all $t \geq 0$.

(iii) $G: \mathbb{C} \rightarrow \mathbb{C}$ is a continuous function.

(iv) $q: \mathbb{R} \rightarrow \mathbb{C}$ is a continuous function satisfying $\sup _{s \in[0,1]} \operatorname{Re}(q(s))<\infty$ and $\overline{q([0,1])} \cap\{\lambda \in \mathbb{C}: \operatorname{Re} \lambda \geq b\}$ is bounded for every $b \in \mathbb{R}$.

(v) $f_{1}:[0,1] \rightarrow \mathbb{R}$ is integrable and $f_{2}: \mathbb{R} \rightarrow \mathbb{R}$ is Lipschitzian with a Lipschitz constant $L_{f_{2}}$.

Remark 4.1. Let $f(t, x)=f_{1}(t) f_{2}(x)$. From assumption (v) we know that

$$
|f(t, x)| \leq\left|f_{1}(t)\right|\left|f_{2}(x)\right| \leq\left|f_{1}(t)\right|\left(\left|f_{2}(0)\right|+L_{f_{2}}|x|\right) \leq\left|f_{1}(t)\right| \Omega(|x|),
$$

where $\Omega(r)=\left|f_{2}(0)\right|+L_{f_{2}} r$. Further, in virtue of Lemma 2.4 we have

$$
\alpha(f(t, D)) \leq\left|f_{1}(t)\right| \alpha\left(f_{2}(D)\right) \leq\left|f_{1}(t)\right| L_{f_{2}} \alpha(D),
$$

for each $D \subset \mathbb{R}$.

We define the multiplication operator $A$ induced on $X$ as follows:

$$
\left\{\begin{array}{l}
D(A)=\{f \in X: q . f \in X\} \\
A f=q . f .
\end{array}\right.
$$

From [13, p. 121] we know that $A$ generates a norm continuous multiplication semigroup $\left(T_{q}(t)\right)_{t \geq 0}$ on $X$ given by

$$
T_{q}(t) f=e^{t q} f \text { for } t \geq 0 \text { and } f \in X .
$$

To allow the abstract formulation of (4.1) we define the operators $B(t): Y \rightarrow$ $X$ as follows:

$$
B(t) f=b(t) A f \quad \text { for } t \geq 0 \text { and } f \in D(A) .
$$


Then (4.1) can be rewritten in the abstract form

$$
\left\{\begin{array}{l}
\frac{d}{d t} u(t)=A u(t)+\int_{0}^{t} B(t-s) u(s) d s+f(t, u(t)) \text { for } t \in[0, a], \\
u(0)=u_{0}+g(u) .
\end{array}\right.
$$

Clearly,

$$
|B(t) y| \leq|b(t) A y| \leq b(t)\|y\|
$$

and

$$
\left|\frac{d}{d t} B(t) y\right| \leq\left|b^{\prime}(t)\left\|A y|\leq| b^{\prime}(t) \mid\right\| y\|\leq b(t)\| y \|\right.
$$

for all $y \in Y$ and all $t \in \mathbb{R}^{+}$. Accordingly, the assumptions (H1)-(H4) hold true. Thus, from Theorem $2.2(4.3)$ has a resolvent operator $(R(t))_{t>0}$ on $X$ which is norm continuous for $t>0$.

Now we assume that $G$ is Lipschitz continuous with Lipshitz constant $L_{g}$ satisfying $M_{a}\left(L_{g}+\left|f_{1}\right|_{1} L_{f_{2}}\right)<1$, where $M_{a}=\sup _{0 \leq t \leq a}|R(t)|$. Now, according to Remark 4.1, the conditions of Theorem 3.3 are satisfied. Thus, we have the following result.

Proposition 4.2. Under the assumptions above, the nonlocal problem (4.1) has at least one mild solution on $[0, a]$.

4.2. Application 2. To illustrate the application of the theoretical results of this work, we consider the following integrodifferential equation:

$$
\left\{\begin{array}{l}
\frac{\partial \omega(t, \xi)}{\partial t}=A \omega(t, \xi)+\int_{0}^{t} \beta e^{-\alpha(t-s)} A \omega(s, \xi) d s+p_{1}(t) p_{2}(\omega(t, \xi)) \\
\text { for } t \in I:=[0, a], \xi \in[0,2 \pi] \\
\omega(t, 0)=\omega(t, 2 \pi), \text { for } t \in I \\
\omega(0, \xi)=\sum_{i=1}^{n} c_{i} \omega\left(t_{i}, \xi\right) \\
\text { for } 0 \leq \xi \leq 2 \pi, t_{1}<t_{2}<\cdots<t_{n}, t_{i} \in I, c_{i} \in \mathbb{R}
\end{array}\right.
$$

where the constants $\alpha, \beta$ satisfy $0 \leq \alpha \leq 1, \beta \geq 0$ and the functions $p_{1}: I \rightarrow \mathbb{R}$ and $p_{2}: X \rightarrow X$ satisfy appropriate conditions which are specified later. The operator $A$ is defined by

$$
A \omega(t, \xi)=a_{1}(\xi) \frac{\partial^{2}}{\partial \xi^{2}} \omega(t, \xi)+b_{1}(\xi) \frac{\partial}{\partial \xi} \omega(t, \xi)+\bar{c}(\xi) \omega(t, \xi),
$$

with domain

$$
D(A)=\left\{v \in L^{2}([0,2 \pi], \mathbb{R}): v^{\prime}, v^{\prime \prime} \in L^{2}([0,2 \pi], \mathbb{R}), v(0)=v(2 \pi)\right\},
$$

where the coefficients $a_{1}, b_{1}$ and $\bar{c}$ are suitably smooth and satisfy the usual uniformly ellipticity condition.

The above problem serves as a model for the the study of heat conduction in materials with memory. We refer to $[25,30]$ for a discussion of the physical interpretation of (4.4) and a much more complete summary of previous related 
work. In [27], the authors combine iterative techniques with the Hausdorff measure of noncompactness and fixed point theory to prove the existence of mild solutions for the semilinear integrodifferential equation (4.4) subject to compact nonlocal initial conditions. Our particular concern in this section will be in obtaining similar results under weaker assumptions and subject to non-compact nonlocal conditions.

To allow the abstract formulation of (4.4), we will simply follow the notation and terminology of [27].

Let $X=L^{2}([0,2 \pi] ; \mathbb{R})$ be the Banach space of square integrable functions from $[0,2 \pi]$ into $\mathbb{R}$ and let $u(t)=\omega(t,$.$) Equation (4.4) may be written$ abstractly in the shape

$$
\left\{\begin{array}{l}
u^{\prime}(t)=A u(t)+\int_{0}^{t} B(t-s) u(s) d s+f(t, u(t)), t \in I, \\
u(0)=g(u),
\end{array}\right.
$$

where, $B(t)=\beta e^{-\alpha t} A$, the function $g: C(I ; X) \rightarrow X$ is given by

$$
g(u)=\sum_{i=1}^{n} c_{i} u\left(t_{i}\right), 0<t_{1}<t_{2}<\cdots<t_{n} \leq a
$$

and the function $f:[0, a] \times X \rightarrow X$ is given by $f(t, x)=p_{1}(t) p_{2}(x)$, here $p_{1}: I \rightarrow \mathbb{R}$ is integrable on $I$, and $p_{2}: X \rightarrow X$ is a Lipschitzian function with a Lipschitz constant $L_{p_{2}}$. The unknown function $u(t)$ takes values in $X$. Note that $g$ is not compact and so the approach used in [27] fails in our considerations. We apply Theorem 3.3 to get the desired result. Notice first that

$$
\left|g\left(u_{1}\right)-g\left(u_{2}\right)\right| \leq\left(\max _{0 \leq i \leq n}\left|c_{i}\right|\right)\left|u_{1}-u_{2}\right| .
$$

From [13] we know that $A$ generates a bounded analytic, noncompact semigroup $(T(t))_{t \geq 0}$ on $X$. Thus, (H1), (H4) and (H5) hold true. Taking into account the fact that the linear operator $A: Y \rightarrow X$ is continuous we infer that $B(t)$ is continuous from $Y$ to $X$ for all $t \geq 0$. Moreover, if we take $b(t):=\beta e^{-\alpha t}$ then $b: \mathbb{R}^{+} \rightarrow \mathbb{R}^{+}$is locally integrable, $\left|b^{\prime}(t)\right| \leq b(t)$ and

$$
|B(t) y| \leq|b(t) A y| \leq b(t)\|y\|
$$

for all $y \in Y$ and $t \geq 0$. Therefore, $(\mathbf{H 2})$ is verified. Notice for any $y \in Y$, the map $t \rightarrow B(t) y$ belongs to $W_{l o c}^{1,1}\left(\mathbb{R}^{+}, X\right)$ and

$$
\left|\frac{d}{d t} B(t) y\right| \leq \alpha b(t)|A y| \leq b(t)\|y\|
$$

for $y \in Y$ and all $t \in \mathbb{R}^{+}$. This shows that (H3) is satisfied. Further, the function $f$ satisfies

$$
|f(t, x)| \leq\left|p_{1}(t)\right|\left|p_{2}(x)\right| \leq\left|p_{1}(t)\right|\left(\left|p_{2}(0)\right|+L_{p_{2}}|x|\right) \leq\left|p_{1}(t)\right| \Omega(|x|),
$$


with $\Omega(r)=\left|p_{2}(0)\right|+L_{p_{2}} r$. Also, for any bounded subset $D$ of $X$ we have

$$
\alpha(f(t, D)) \leq\left|p_{1}(t)\right| \alpha\left(p_{2}(D)\right) \leq\left|p_{1}(t)\right| L_{p_{2}} \alpha((D)) .
$$

Thus, (H7) and (H8) are satisfied. Finally, notice

$$
\lim _{r \rightarrow \infty} \frac{\Omega(r)}{r}=L_{p_{2}} \text {. }
$$

Thus, the conditions of Theorem 3.3 are fulfilled whenever

$$
M_{a}\left|p_{1}\right|_{1} L_{p_{2}}+\max _{0 \leq i \leq n}\left|c_{i}\right| M_{a}<1,
$$

and therefore Equation (4.4) has a mild solution.

REMARK 4.3. In the application above we have considered a simple nonlinear integrodifferential equation from heat conduction in materials with memory. However, the approach developed in this work can be applied in a similar way to a large class of integrodifferential equations of the form (1.1), when the compactness of the semigroup or the nonlocal condition fails to hold.

\section{Concluding Remarks}

In the present work, we deal with the existence of continuous solutions for a class of nonlinear partial integrodifferential equations with nonlocal conditions. The condition assumed in this work for the nonlocal term $g$ is of a fairly simple nature. It is important to note that we do not require the compactness of $g$. Furthermore, the immediate norm continuity assumption we used for the strongly continuous semigroup $T(t)$ is more practical than the immediate norm continuity of the resolvent operator assumed by other authors. However, the authors conjecture that this assumption is redundant and can be dropped. Only the fact that $A$ generates a strongly continuous semigroup is needed.

ACKNOWLEDGEMENTS.

The authors would like to thank the referees for very deep and useful comments and suggestions that contributed to the improvement of the original version of this work.

\section{REFERENCES}

[1] J. Banaś and K. Goebel, Mesure of noncompactness in Banach spaces, Marcel Dekker, New York, 1980.

[2] D. Bothe, Multivalued perturbations of m-accretive differential inclusions, Israel J. Math. 108 (1998), 109-138.

[3] L. Byszewski, Theorems about the existence and uniqueness of solutions of a semilinear evolution nonlocal Cauchy problem, J. Math. Anal. Appl. 162 (1991), 494-505.

[4] L. Byszewski, Existence and uniqueness of solutions of semilinear evolution nonlocal Cauchy problem, Zeszyty Nauk. Politech. Rzeszowskiej Mat. Fiz. 18 (1993), 109-112. 
[5] L. Byszewski and V. Lakshmikantham, Theorem about the existence and uniqueness of a solution of a nonlocal abstract Cauchy problem in a Banach space, Appl. Anal. 40 (1991), 11-19.

[6] L. Byszewski and H. Akca, Existence of solutions of a semilinear functionaldifferential evolution nonlocal problem, Nonlinear Anal. 34 (1998), 65-72.

[7] P. Cannarsan and D. Sforza, Global solutions of abstract semilinear parabolic equations with memory terms, NoDEA Nonlinear Differential Equations Appl. 10 (2003), 399430 .

[8] G. Chen and R. Grimmer, Semigroups and integral equations, J. Integral Equations 2 (1980), 133-154.

[9] E. N. Chukwu, Differential models and neutral systems for controlling the wealth of nations, World Scientific Publishing, River Edge, 2001.

[10] W. Desch, R. Grimmer and W. Schappacher, Some considerations for linear integrodifferential equations, J. Math. Anal. Appl. 104 (1984), 219-234.

[11] K. Deng, Exponential decay of solutions of semilinear parabolic equations with nonlocal initial conditions, J. Math. Anal. Appl. 179 (1993), 630-637.

[12] J. P. C. Dos Santos, S. M. Guzzo and M. N. Rabelo, Asymptotically almost periodic solutions for abstract partial neutral integro-differential equation, Adv. Difference Equ. 2010, Art. ID 310951, 26 pp.

[13] K.-J. Engel and R. Nagel, One-parameter semigroups for linear evolution equations, Springer-Verlag, New York, 2000.

[14] K. Ezzinbi and J. H. Liu, Nondensely defined evolution equations with nonlocal conditions, Math. Comput. Modelling 36 (2002), 1027-1038.

[15] K. Ezzinbi, S. Ghnimi and M. A. Taoudi, Existence and regularity of solutions for neutral partial functional integrodifferential equations with infinite delay, Nonlinear Anal. Hybrid Syst. 4 (2010), 54-64.

[16] K. Ezzinbi and X. Fu, Existence and regularity of solutions for some neutral partial differential equations with nonlocal conditions, Nonlinear Anal. 57 (2004), 1029-1041.

[17] K. Ezzinbi, X. Fu and K. Hilal, Existence and regularity in the $\alpha$-norm for some neutral partial differential equations with nonlocal conditions, Nonlinear Anal. 67 (2007), 1613-1622.

[18] K. Ezzinbi and M. A. Taoudi, Sadovskii-Krasnosel'skii type fixed point theorems in Banach spaces with application to evolution equations, J. Appl. Math. Comput. 49 (2015), 243-260.

[19] X. Fu and K. Ezzinbi, Existence of solutions for neutral functional differential evolution equations with nonlocal conditions, Nonlinear Anal. 54 (2003), 215-227.

[20] R. C. Grimmer, Resolvent operators for integral equations in a Banach space, Trans. Amer. Math. Soc. 273 (1982), 333-349.

[21] G. Gripenberg, S.-O. Londen and O. Staffans, Volterra integral and functional equations, Cambridge University Press, Cambridge, 1990.

[22] M. E. Gurtin and A. C. Pipkin, A general theory of heat conduction with finite wave speed, Arch. Rational Mech. Anal. 31 (1968), 113-126.

[23] E. Hernández, M. Pierri and A. Prokopczyk, On a class of abstract neutral functional differential equations, Nonlinear Anal. 74 (2011), 3633-3643.

[24] J. Liang, J. Liu and T. J. Xiao, Nonlocal Cauchy problems governed by compact operator families, Nonlinear Anal. 57 (2004), 183-189.

[25] J. Liang and T. J. Xiao, Semilinear integrodifferential equations with nonlocal initial conditions, Comput. Math. Appl. 47 (2004), 863-875.

[26] L. Liu, F. Guo, C. Wu and Y. Wu, Existence theorems of global solutions for nonlinear Volterra type integral equations in Banach spaces, J. Math. Anal. Appl. 309 (2005), 638-649. 
[27] C. Lizama and J. C. Pozo, Existence of mild solutions for a semilinear integrodifferential equation with nonlocal initial conditions, Abstr. Appl. Anal. 2012 (2012), Art. ID $647103,15 \mathrm{pp}$.

[28] A. Lunardi, On the linear heat equation with fading memory, SIAM J. Math. Anal. 21 (1990), 1213-1224.

[29] R. C. MacCamy, An integro-differential equation with application in heat flow, Quart. Appl. Math. 35 (1977/78), 1-19.

[30] R. K. Miller, An integro-differential equation for rigid heat conductors with memory, J. Math. Anal. Appl. 66 (1978), 313-332.

[31] H. Monch, Boundary value problems for nonlinear ordinary differential equations of second order in Banach spaces, Nonlinear Anal. 4 (1980), 985-999.

[32] S. Ntouyas and P. Tsamotas, Global existence for semilinear evolution equations with nonlocal conditions, J. Math. Anal. Appl. 210 (1997), 679-687.

[33] J. W. Nunziato, On heat conduction in materials with memory, Quart. Appl. Math. 29 (1971), 187-204.

[34] J. Pruss, Evolutionary integral equations and applications, Birkhäuser, Basel, 1993.

[35] J. Sun and X. Zhang, A fixed point theorem for convex-power condensing operators and its applications to abstract semilinear evolution equations, Acta Math. Sinica (Chin. Ser.) 48 (2005), 439-446.

[36] L. Zhu and G. Li, Existence results of semilinear differential equations with nonlocal initial conditions in Banach spaces, Nonlinear Anal. 74 (2011), 5133-5140.

K. Ezzinbi

Department of Mathematics, Faculty of Sciences Semlalia

Cadi Ayyad University

BP 2390 Marrakech

Morocco

E-mail: ezzinbi@uca.ma

\section{S. Ghnimi}

Department of Mathematics, Faculty of Sciences

University of Gafsa

B.P. 2112 Gafsa

Tunisia

E-mail: ghnimisaifeddine@yahoo.fr

M.-A. Taoudi

Cadi Ayyad University

National School of Applied Sciences

Marrakech

Morocco

E-mail: a.taoudi@uca.ma

Received: 31.1.2015.

Revised: 19.11.2015. 